\title{
APLICACIÓN DE LAS PROPIEDADES NUTRACÉUTICASY ESTABILIZANTES DEL MESOCARPIO Y EXOCARPIO DEL MARACUYÁ EN LA OBTENCIÓN DE UNA BEBIDA NATURAL DE CHINOLA-PIÑA
}

\section{Application of the nutraceutical and stabilizing properties of the passion fruit mesocarp and exocarp in obtaining a natural passion fruit-pineapple drink}

\section{Dileisys A. Paniaguaa, Katiuska M. Vicioso ${ }^{\mathrm{b}}$ y Niover G. Valdez}

Recibido: 6 de mayo, 2020 • Aprobado: 26 de julio, 2020

Cómo citar: Paniagua DA, Vicioso KM, Valdez NG. Aplicación de las propiedades nutracéuticas y estabilizantes del mesocarpio y exocarpio del maracuyá en la obtención de una bebida natural de chinola-piña. cysa [Internet]. 8 de junio de 2021 [citado 16 de junio de 2021];5(2):7-18. Disponible en: https://revistas.intec.edu.do/index.php/cisa/article/view/2207

\section{Resumen}

Introducción: este estudio consistió en el desarrollo de una bebida natural de maracuyá-pińa, utilizando el exocarpio, mesocarpio y endocarpio del maracuyá como estabilizante y enriquecedor nutritivo de la bebida, convirtiéndolo así en un producto nutracéutico. El mesocarpio del maracuyá puede ser aprovechado por los macronutrientes y micronutrientes que contiene, por sus pigmentos, y por el contenido de varios nutrientes con propiedades terapéuticas.

Material y método: para lograr este objetivo se prepararon pulpas especiales tratadas con biomoléculas que permiten desagregar el material vegetal del cual están compuestas las frutas dejando disponible todos los nutrientes que contiene.

Resultado: de este proceso se obtuvo un jugo estable en su turbidez con un color y olor bastante acentuado, relacionado con las frutas que fueron utilizadas en la investigación. Además, se obtuvo un jugo en donde los nutrientes y moléculas bioactivas atrapadas en el mesocarpio y en el pericarpio de la fruta ahora están disponibles para su inmediata absorción en el organismo humano.

\footnotetext{
a Universidad Autónoma de Santo Domingo, República Dominicana. ORCID: 0000-0002-6877-987X, Correo-e: dpaniagua64@uasd.edu.do
}

\begin{abstract}
Introduction: This study consisted in the development of a natural passion fruit-pineapple drink, taking advantage of the exocarp, mesocarp and endocarp of the passion fruit in the process as stabilizer and nutritive enrichment of the drink, thus turning it into a nutraceutical product. The mesocarp of the passion fruit can be used for the macronutrients and micronutrients it contains, for its pigments, and for the content of various nutrients with therapeutic properties.
\end{abstract}

Material and methods: To achieve this objective, special pulps treated with biomolecules were prepared that allow the disaggregation of the plant material of which the fruits are composed, leaving all the nutrients it contains available.

Result: As a result of this process, a stable juice was obtained in its turbidity with a fairly accentuated color and smell, related to the fruits that were used in the research. In addition, a juice was obtained where the nutrients and bioactive molecules trapped in the mesocarp and in the pericarp

\footnotetext{
b Universidad Autónoma de Santo Domingo, República Dominicana.

${ }^{\text {c } U n i v e r s i d a d ~ A u t o ́ n o m a ~ d e ~ S a n t o ~ D o m i n g o, ~ R e p u ́ b l i c a ~ D o m i n i c a n a . ~}$
} 
Conclusión: el producto tratado con biocatalizador presentó mayor estabilidad y mayores propiedades nutracéuticas que el jugo sin ese tratamiento.

Palabras clave: maracuyá; piña; nutracéutico; mesocarpio; endocarpio.

\section{Introducción}

El maracuyá (Passiflora edulis var. flavicarpa), también conocida como chinola o la fruta de la pasión, es una fruta tropical que pertenece a la familia de Passifloraceae, oriunda de Brasil. Es una enredadora cuyos tallos se trepan en espaldera o enramada, si son conducidos hasta ellas, las hojas son de bordes aserrados alternas con tres lóbulos, de color verde oscuro en el lado superior, las flores están compuestas de sépalos y pétalos amarillentos, y las porciones de la corona delgadas y onduladas, con la mitad inferior morado y la superior blanco-cremosa.

Al contrario de lo que se cree, los beneficios de esta fruta tropical no están escondidos solamente en su pulpa; su cáscara, semillas e incluso hojas son muy beneficiosas para la salud en general ${ }^{1}$. La cáscara evita los picos de insulina, peligrosos para los diabéticos ${ }^{2}$. Contiene nutrientes como proteínas, grasas, carbohidratos, agua, fibra ${ }^{3}$, calcio, fósforo, hierro, tiamina, riboflavina, niacina, ácido ascórbico, retinol, sodio, potasio y varias sustancias con propiedades antioxidantes $^{3,4,5,6}$. Es laxante, ayuda al buen funcionamiento del intestino ${ }^{7,8}$ y disminuye los niveles de colesterol'?.

La piña, ananá o naná (Ananas comosus), es una fruta tropical. Aunque la pińa es originaria de Brasil y Paraguay, hoy en día su consumo está muy extendido por todo el mundo gracias a su delicioso sabor y a las propiedades nutricionales que contiene. Tiene forma ovalada, una corona y también una piel muy rugosa de color verdosa que se va volviendo amarilla y marrón a medida que la piña va madurando, y of the fruit are now available for immediate absorption in the human body. In conclusion, the product treated with a biocatalyst presented greater stability and higher nutraceutical properties than the juice without this treatment.

Keywords: passion fruit; pineapple; nutraceutical; mesocarp; endocarp.

desprende un agradable olor dulzón cuando ya está listo para comer. Por dentro tiene una carne amarilla muy dulce y jugosa. Posee retinol, vitaminas del grupo $\mathrm{B}$ y ácido ascórbico, haciendo de la piña una excelente fuente de antioxidantes que combaten los radicales libres y retrasan el envejecimiento. Hasta un $85 \%$ de la pińa es agua, esto quiere decir que te saciará, te mantendrá bien hidratado y aporta muy pocas calorías (alrededor de unas 55 por cada 100 gramos) ${ }^{10}$.

El propósito del presente estudio es la elaboración de un jugo natural de maracuyá-piña adicionándole las propiedades nutracéuticas y estabilizantes contenidas en el mesocarpio y exocarpio del maracuyá ${ }^{11,15}$.

En el proceso productivo, los cambios de temperatura, los ingredientes utilizados, la presencia de partículas y hasta el transporte y almacenamiento, pueden ser factores que favorezcan la inestabilidad de los sistemas de bebidas, ocasionando la desestabilización de estas, con ello, la formación de precipitados gelatinosos, sedimentación y separación de fases, pérdida de viscosidad e inclusive, turbidez indeseada.

Algunos ingredientes proporcionan una mejor estabilización, promoviendo una apariencia limpia y uniforme, evitando la separación de fases. Entre los estabilizantes que pueden ser utilizados para bebidas están las pectinas. Estas son productos químicos que se obtienen de materias primas vegetales, principalmente frutas, y se usan en varias industrias, en especial la de alimentos, para darle propiedades de gel a los productos; también son utilizadas como estabilizantes. ${ }^{11-15}$ 


\section{Materiales y métodos}

Se realizaron diferentes pruebas de desagregación, la primera y segunda prueba se desarrollaron con agua y mesocarpio del maracuyá, buscando determinar hasta qué cantidad de mesocarpio del maracuyá se puede integrar en agua en términos porcentuales; la segunda prueba fue para establecer los parámetros del proceso. Se procedió a calentar en un beaker 2,000 $\mathrm{mL}$ de agua entre $50-55^{\circ} \mathrm{C}$. Se pesaron diferentes porcentajes del mesocarpio del maracuyá y se desagregó en 500 mL de agua. Se ajustó el pH aproximadamente entre 4.5-4.8 con un álcali o un ácido diluido según fuese necesario. Luego se le adicionó el catalizador y se colocó en un plato rotatorio calorífico a la misma temperatura inicial.

Las siguientes cuatro pruebas de desagregación se realizaron utilizando jugo de pińa y mesocarpio del maracuyá, con el objetivo de determinar cuál de las concentraciones del mesocarpio del maracuyá era la adecuada, aplicando el jugo de pińa como base, se hicieron los ajustes de lugar, obteniendo un producto final con contenido de fibras de tamaño más o menos uniforme, lo más pequeño posible y mejorando las características organolépticas. Se procedió a llevar aproximadamente a $40{ }^{\circ} \mathrm{C}$ una porción de jugo de pińa y a agregar el catalizador. Se desagregaron tres porcentajes diferentes del mesocarpio del maracuyá en el jugo de pińa, adicionando el catalizador y verificando el resultado de los parámetros. Se procedió a colocar en un beaker, jugo de piña y se llevó a $35^{\circ} \mathrm{C}$ y luego se agregó el catalizador. Se pesó entre 15-20\% del mesocarpio del maracuyá y se desagregó en diferentes porciones, tomando en cuenta el tiempo de desagregación de cada parte y midiendo los parámetros de control antes de cada desagregación, filtrando y realizando pruebas con diferentes saborizantes naturales.

Las pruebas de desagregación siguientes, fueron con el mesocarpio y placenta del maracuyá, en jugo de pińa, con alimentación continua. Se procedió a calentar a $35^{\circ} \mathrm{C}$ una cantidad determinada de jugo de piña y a adicionar el catalizador. Se desagregó entre un 5-10\% de la placenta de la chinola y se filtró. Se midió la cantidad obtenida, se desagregó entre un 20-25\% del mesocarpio de la chinola en porciones de igual tamańo, tomando en cuenta el tiempo de degradación de cada porción, midiendo los parámetros de controles luego de cada alimentación y al final filtrar. Se realizaron las diluciones necesarias para obtener el ${ }^{\circ}$ Brix deseado y ajustando acidez al producto obtenido con diferentes acidulantes naturales.

Para mejorar las características organolépticas al producto obtenido en las pruebas anteriores de desagregación, se procedió a realizar una mezcla de jugo de piña y jugo de maracuyá, se calentó a $35^{\circ} \mathrm{C}$ una cantidad determinada de jugo de piña y se adicionó el catalizador. Se agregó entre un 5-10 \% de placenta de maracuyá, se filtró y midió la cantidad obtenida. Al producto obtenido, se le adicionó entre un 5-10 $\%$ de mesocarpio del maracuyá y se desagregó en porciones iguales, tomando en cuenta el tiempo de desagregación, filtrando y midiendo los parámetros de control. Realizando los ajustes necesarios para obtener los grados brix deseados.

En la caracterización del producto se determinó proteína cruda [AOAC 2001.11], grasa cruda [AOAC 920.39], fibra cruda [AOAC 962.09], ceniza [AOAC 942.05], acidez [AOAC 942.15], sólidos solubles [AOAC 983.17] y el pH [AOAC 971,27]. ${ }^{16}$

Para la determinación de la actividad enzimática se utiliza el método del papel de filtro ${ }^{21}$.

Para determinar la cinética de la celulasa, se preparó una muestra al $30 \%$ del mesocarpio del maracuyá en una mezcla de jugo chinola piña. Se ajustó el pH a 4.8 con un álcali. Se llevó la muestra a una temperatura entre $42-50{ }^{\circ} \mathrm{C}$ en bańo de agua, con el fin de trabajar con el catalizador en condiciones óptimas. Se agregó a la muestra el catalizador y se mantuvo en agitación constante, se muestreó por duplicado 
cada 10 minutos y se centrifugó. Del centrifugado se tomaron alícuotas de $0.5 \mathrm{~mL}$ y se continuó el procedimiento como en actividad enzimática ${ }^{17}$.

Para determinar la estabilidad del producto, se sometió la muestra, recién preparada, a diferentes temperaturas y al mismo tiempo se realizó una mezcla de pulpa de pińa y maracuyá simple, para usarla como comparativo. Se realizó la prueba por duplicado y se utilizaron botellas de aproximadamente 12 onzas, de cuello corto, transparentes e incoloras. Se observaron las muestras y a su vez se midió la sedimentación en centímetros. Se midió la sedimentación a la 5 ta hora de la elaboración de las muestras y luego a las 24 y 48 horas, respectivamente.

Las temperaturas de las áreas a las que las muestras fueron sometidas son:

Área 1: $\pm 5^{\circ} \mathrm{C}$ Área 2: $\pm 17^{\circ} \mathrm{C}$ Área 3: $\pm 25^{\circ} \mathrm{C}$ Área 4: $\pm 30^{\circ} \mathrm{C}$

\section{Resultados}

\section{Determinación actividad enzimática de la Celulasa}

Se midieron las absorbancias del estándar de glucosa y los resultados fueron los siguientes:

Tabla 1. Resultados curva calibración Glucosa

\begin{tabular}{|c|c|}
\hline $\begin{array}{c}\text { Concentración } \\
\text { Glucosa }(\mathbf{m g} / \mathbf{m L})\end{array}$ & Absorbancia \\
\hline 0.0 & 0.00 \\
\hline 2.4 & 0.142 \\
\hline 8.8 & 0.517 \\
\hline 12.0 & 0.687 \\
\hline 15.6 & 0.858 \\
\hline
\end{tabular}

Fuente: elaboración propia.

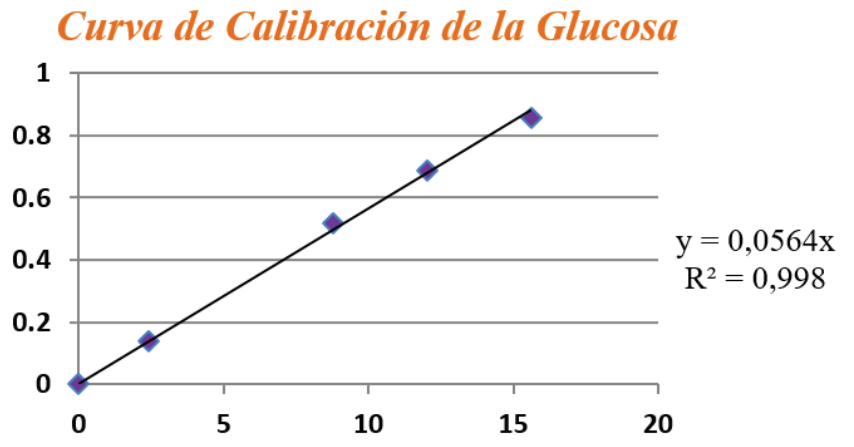

Concentración Glucosa $(\mathrm{mg} / \mathrm{mL})$

Figura 1. Curva de Calibración de la Glucosa Fuente: datos tabla 1.

\section{Cálculo de la actividad enzimática}

Tabla 2. Resultados concentraciones y absorbancias actividad enzimática

\begin{tabular}{|c|c|}
\hline Concentración & Absorbancia \\
\hline 14.523 & 0.822 \\
\hline 14.735 & 0.834 \\
\hline 14.894 & 0.843 \\
\hline $\mathrm{X}=14.717$ & $\mathrm{X}=0.833$ \\
\hline
\end{tabular}

Fuente: elaboración propia.

$\mathbf{A u}=\frac{\text { Miligramos de glucosa producida }}{\text { M glucosa } \cdot \text { tiempo } \cdot \mathrm{mL} \text { catalizador }}$

M glucosa: $180 \mathrm{mg} / \mathrm{mmol}$

Tiempo: 60 minutos

$\mathrm{Au}=$

$147.2 \mathrm{mg}$

\section{$180 \mathrm{mg} / \mathrm{mmol}$ X $60 \mathrm{~min} \times 0.5 \mathrm{~mL}$}

\section{$\mathrm{Au}=0.03 \mathrm{mmol} \cdot \mathrm{min}^{-1} \cdot \mathrm{mL}^{-1}$}

\section{Cinética en el jugo con tratamiento}

Para presentar los resultados obtenidos en la cinética, se construyó un gráfico Absorbancia en función del tiempo. 
Aplicación de las propiedades nutracéuticas y estabilizantes del mesocarpio y exocarpio del maracuyá en la obtención de una bebida natural de chinola-piña

Tabla 3. Resultados de la Cinética Enzimática

\begin{tabular}{|c|c|c|c|c|c|c|c|c|c|c|c|c|c|c|}
\hline Tiempo (min) & 0 & 5 & 15 & 20 & 25 & 35 & 45 & 55 & 65 & 75 & 85 & 95 & 105 & 115 \\
\hline Absorbancia & 0.037 & 0.056 & 0.074 & 0.085 & 0.100 & 0.100 & 0.115 & 0.115 & 0.123 & 0.129 & 0.130 & 0.130 & 0.130 & 0.130 \\
\hline
\end{tabular}

Fuente: elaboración propia. Resultado de la investigación

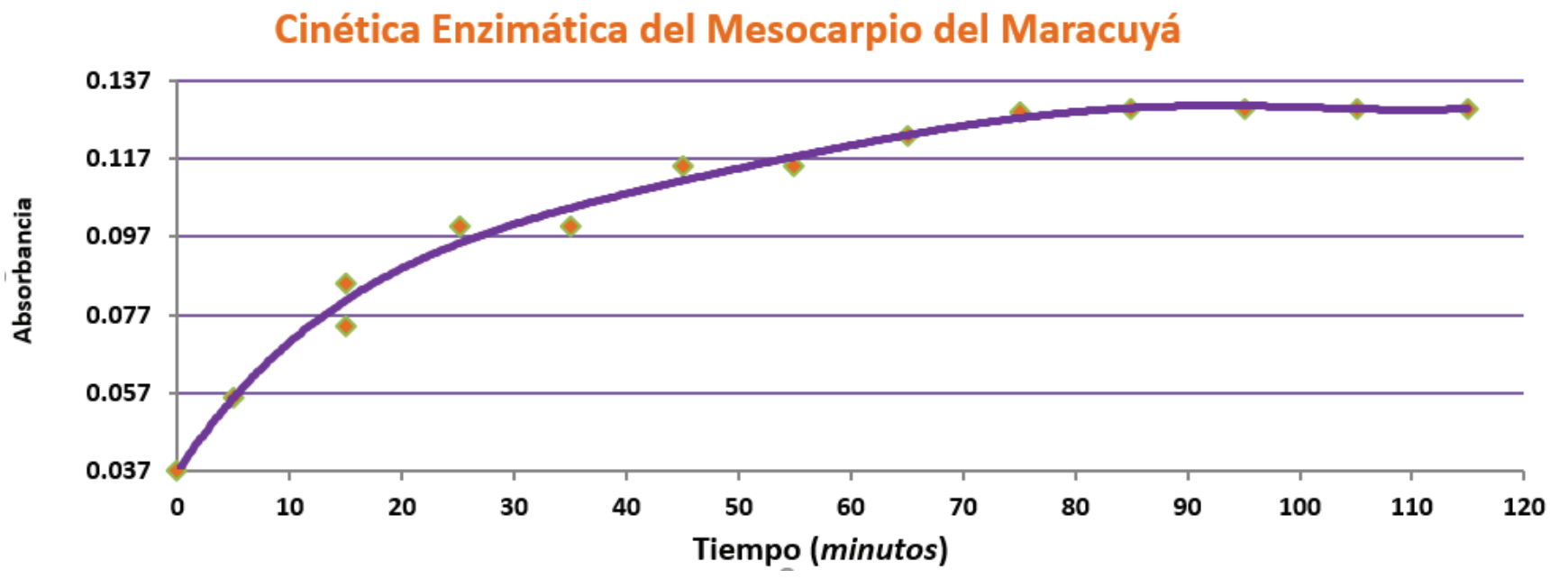

Figura 2. Cinética Enzimática del Mesocarpio del Maracuyá

Fuente: datos tabla 3. Resultado de la investigación

Datos de los parámetros fisicoquímicos y contenido de macronutrientes de jugo pińa, jugo maracuyá, jugo pińa-maracuyá y jugo con tratamiento

Tabla 4. Resultados de las pruebas físico-química jugos

\begin{tabular}{|c|c|c|c|c|}
\hline Parámetros & Jugo Pińa & Jugo Maracuyá & $\begin{array}{c}\text { Pina-Maracuyá } \\
\mathbf{7 5 : 2 5}\end{array}$ & $\begin{array}{c}\text { Jugo con trata- } \\
\text { miento }\end{array}$ \\
\hline Proteína bruta & 0.41 & 0.87 & 0.53 & 0.85 \\
\hline Grasa bruta & 3.16 & 2.73 & 3.05 & 3.44 \\
\hline Carbohidratos digeribles & 5.31 & 7.53 & 5.86 & 4.56 \\
\hline Fibra cruda & 0.04 & 0.06 & 0.05 & 0.28 \\
\hline Cenizas & 0.21 & 0.62 & 0.28 & 0.33 \\
\hline Sólidos solubles $\left({ }^{\circ} \mathrm{Brix}\right)$ & $14.0^{\circ}$ & 13.2 & $13.6^{\circ}$ & $12.7^{\circ}$ \\
\hline$\%$ Acidez & $0.51 \%$ & $0.44 \%$ & $0.49 \%$ & $0.31 \%$ \\
\hline $\mathrm{pH}$ & 3.50 & & 3.30 & 3.50 \\
\hline
\end{tabular}

Fuente: elaboración propia. Resultados de la investigación 
Determinación de la estabilidad de la muestra con tratamiento, en términos comparativos con una muestra de mezcla de jugo simple (sin tratamiento)

Tabla 5. Determinación Estabilidad por sedimentación

\begin{tabular}{|l|c|c|}
\hline \multicolumn{3}{|c|}{ Sedimentación $(\mathbf{c m})$ a la 5ta hora } \\
\hline Áreas & Jugo sin tratamiento & Jugo con tratamiento \\
\hline Área $1\left( \pm 5^{\circ} \mathrm{C}\right)$ & $1.5 \mathrm{~cm}$ & $0.1 \mathrm{~cm}$ \\
\hline Área $2\left( \pm 17^{\circ} \mathrm{C}\right)$ & $2.5 \mathrm{~cm}$ & $0.3 \mathrm{~cm}$ \\
\hline Área $3\left( \pm 25^{\circ} \mathrm{C}\right)$ & $3.0 \mathrm{~cm}$ & $0.4 \mathrm{~cm}$ \\
\hline Área $4\left( \pm 30^{\circ} \mathrm{C}\right)$ & $5.6 \mathrm{~cm}$ & $0.5 \mathrm{~cm}$ \\
\hline
\end{tabular}

Fuente: elaboración propia. Resultados de la investigación

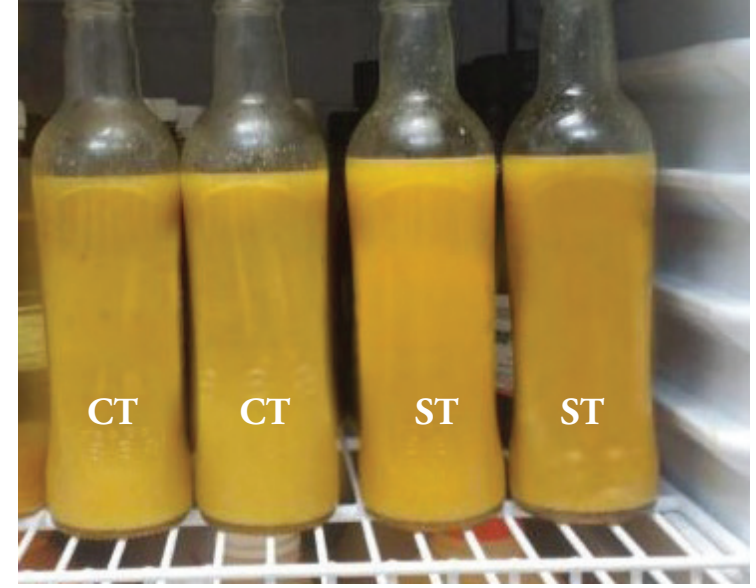

Figura 3. Sedimentación 5 hora en el área 1

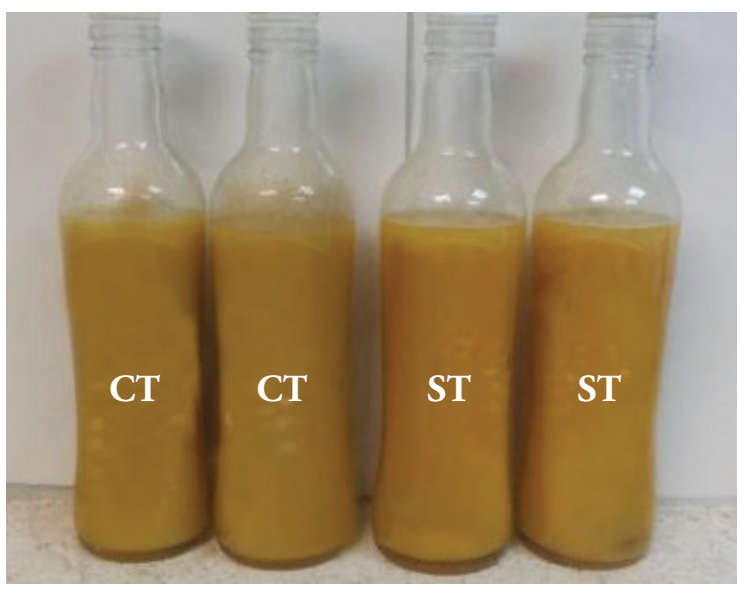

Figura 5. Sedimentación 5 hora en el área 3

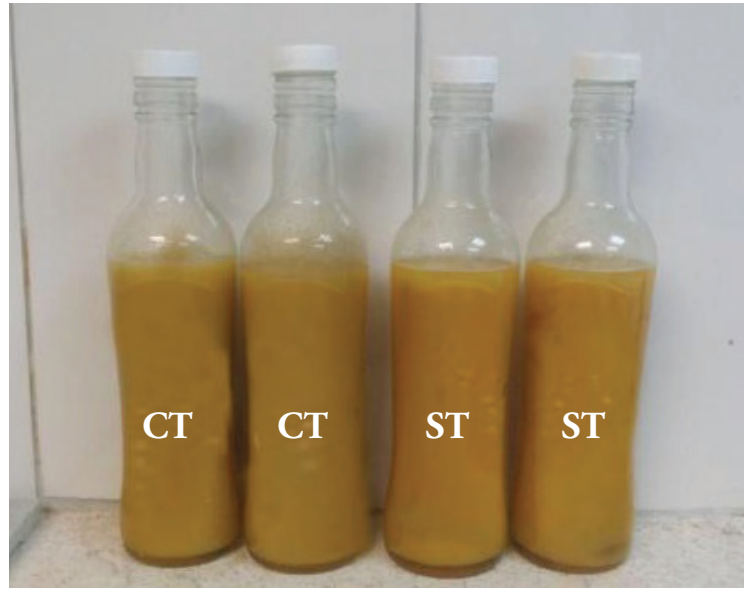

Figura 4. Sedimentación 5 hora en el área 2

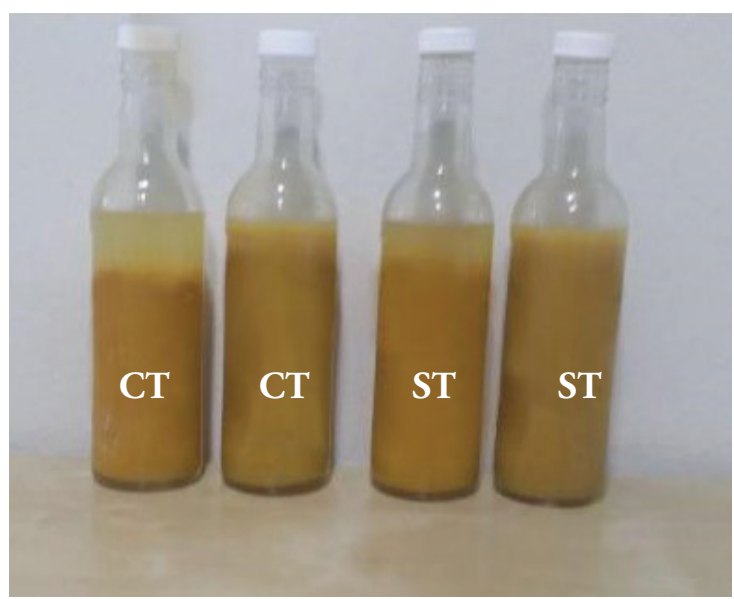

Figura 6. Sedimentación 5 hora en el área 4

${ }^{*}$ Con tratamiento $(C T)$ y sin tratamiento $(S T)$ con biomoléculas. 
Aplicación de las propiedades nutracéuticas y estabilizantes del mesocarpio y exocarpio del maracuyá en la obtención de una bebida natural de chinola-piña

Tabla 6. Determinación Estabilidad por sedimentación

\begin{tabular}{|l|c|c|}
\hline \multicolumn{3}{|c|}{ Sedimentación $(\mathbf{c m})$ a las 24 horas } \\
\hline Áreas & Jugo sin tratamiento & Jugo con tratamiento \\
\hline Área $1\left( \pm 5^{\circ} \mathrm{C}\right)$ & $2.3 \mathrm{~cm}$ & $0.2 \mathrm{~cm}$ \\
\hline Área $2\left( \pm 17^{\circ} \mathrm{C}\right)$ & $4.4 \mathrm{~cm}$ & $0.4 \mathrm{~cm}$ \\
\hline Área $3\left( \pm 25^{\circ} \mathrm{C}\right)$ & $4.9 \mathrm{~cm}$ & $3.8 \mathrm{~cm}$ \\
\hline Área $4\left( \pm 30^{\circ} \mathrm{C}\right)$ & $5.2 \mathrm{~cm}$ & $4.3 \mathrm{~cm}$ \\
\hline
\end{tabular}

Fuente: elaboración propia. Resultados de la investigación

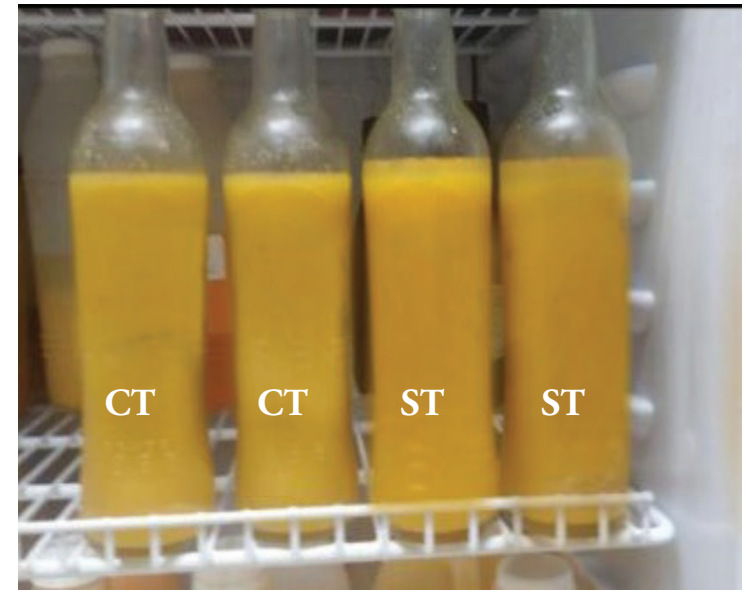

Figura 7. Sedimentación 24 horas en el área 1

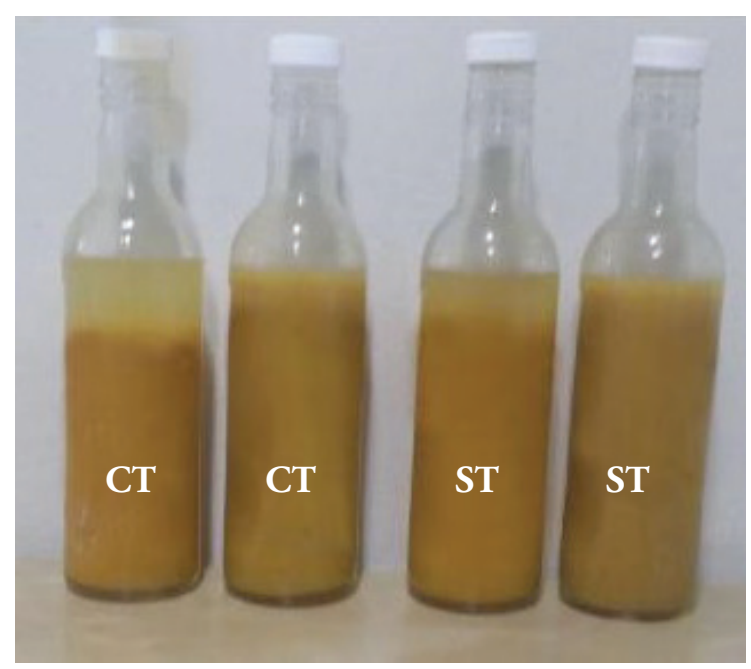

Figura 9. Sedimentación 24 horas en el área 3

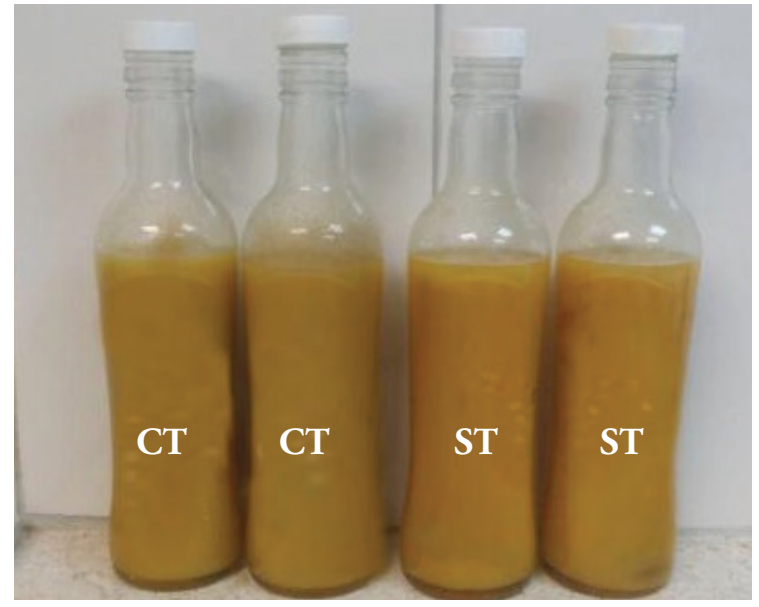

Figura 8. Sedimentación 24 horas en el área 2

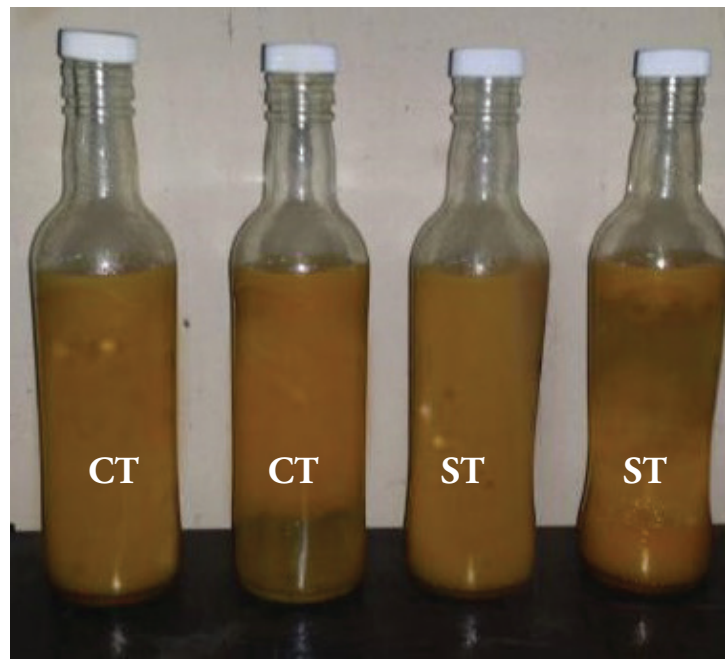

Figura 10. Sedimentación 24 horas en el área 4

*Con tratamiento (CT) y sin tratamiento (ST) con biomoléculas. 
Tabla 7. Determinación Estabilidad por sedimentación

\begin{tabular}{|c|c|c|}
\hline \multicolumn{3}{|c|}{ Sedimentación $(\mathbf{c m})$ a las 48 horas } \\
\hline Áreas & Jugo sin tratamiento & Jugo con tratamiento \\
\hline Área $1\left( \pm 5^{\circ} \mathrm{C}\right)$ & $2.2 \mathrm{~cm}$ & $0.2 \mathrm{~cm}$ \\
\hline Área $2\left( \pm 17^{\circ} \mathrm{C}\right)$ & $5.1 \mathrm{~cm}$ & $5.0 \mathrm{~cm}$ \\
\hline Área $3\left( \pm 25^{\circ} \mathrm{C}\right)$ & $5.3 \mathrm{~cm}$ & $5.2 \mathrm{~cm}$ \\
\hline Área $4\left( \pm 30^{\circ} \mathrm{C}\right)$ & $5.8 \mathrm{~cm}$ & $5.5 \mathrm{~cm}$ \\
\hline
\end{tabular}

Fuente: elaboración propia. Resultados de la investigación

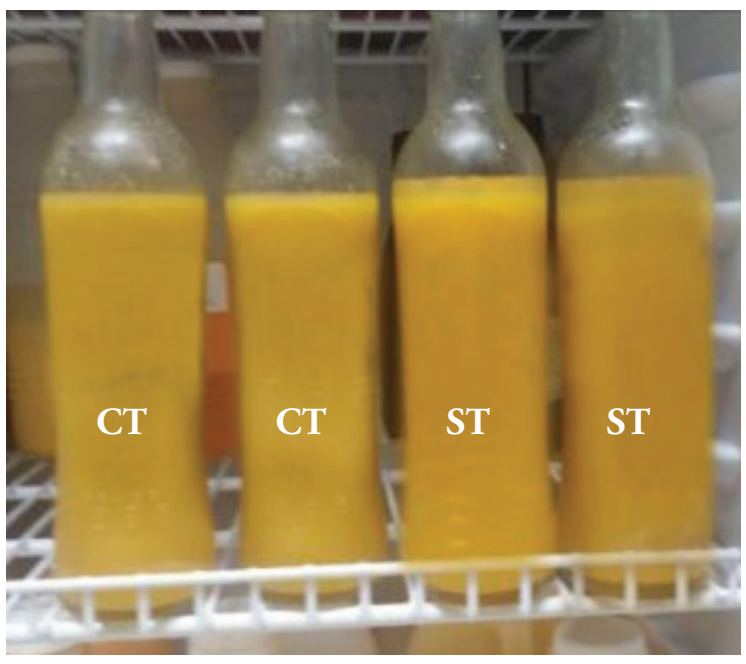

Figura 11. Sedimentación 48 horas en el área 1

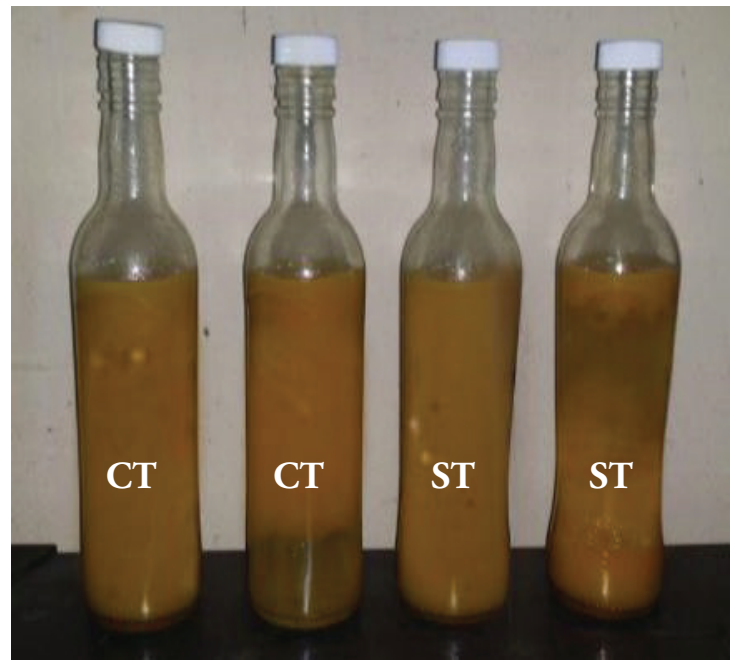

Figura 13. Sedimentación 48 horas en el área 3

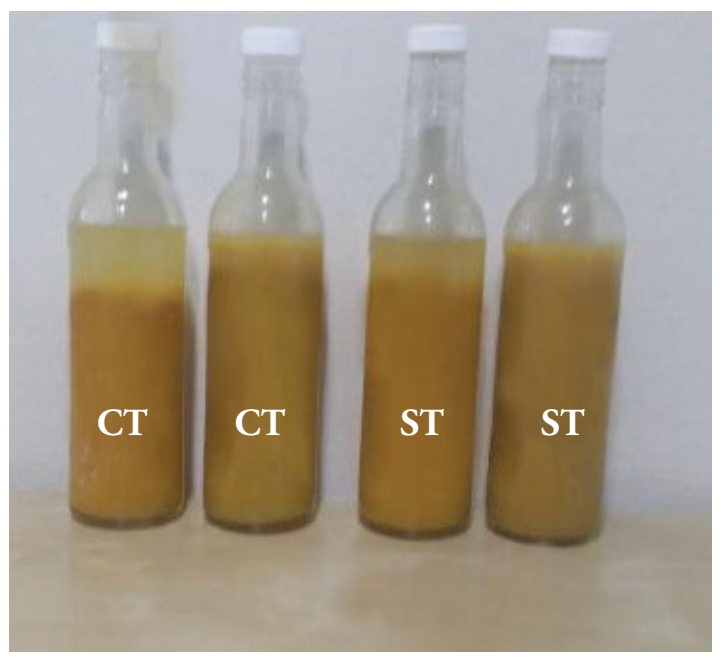

Figura 12. Sedimentación 48 horas en el área 2

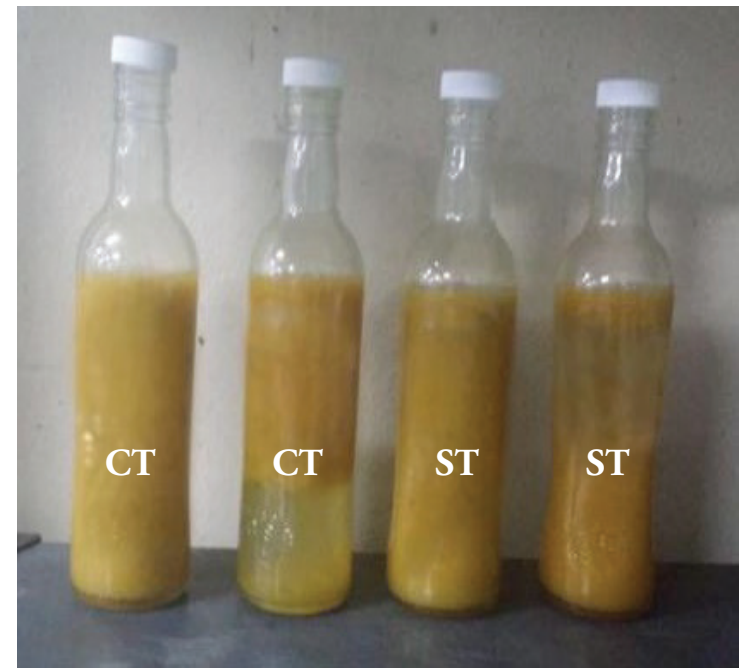

Figura 14. Sedimentación 48 horas en el área 4

*Con tratamiento (CT) y sin tratamiento (ST) con biomoléculas 


\section{Discusión}

Para obtener este producto y viabilizar la transferencia de los resultados a la industria se establecieron los parámetros de la actividad y cinética enzimática, ambos son muy importantes debido a que permiten determinar la relación tiempo de reacción-cantidad del catalizador a utilizar vs. costo del proceso. Otros datos importantes que involucran este proceso son la cantidad de biocatalizador a utilizar y parámetros óptimos de la reacción. Bajo ciertas condiciones como $\mathrm{pH}$, concentración de sustrato y temperatura, el proceso de desagregación del mesocarpio y exocarpio de la chinola es de 75 minutos, tal como se observa en el plato del gráfico de la figura 2.

Para trabajar con el catalizador en su óptimo nivel, se deben tomar en cuenta la temperatura y el $\mathrm{pH}$ a la que este estará sometido. Estos parámetros en la muestra juegan un papel muy importante, debido a que, si se trabaja fuera de los rangos óptimos del catalizador, este se puede inactivar. Con esta prueba se logró determinar que el catalizador actúa sobre la celulosa contenida en el mesocarpio del maracuyá, desagregando los azúcares que contiene. En el proceso también se obtuvo un producto en donde los nutrientes y moléculas bioactivas atrapadas en el mesocarpio y exocarpio de la fruta ahora están disponibles para su inmediata absorción en el organismo humano.

Los resultados de los análisis, a lo que fueron sometido los productos obtenidos, evidencian que el tratamiento catalítico natural con biomoléculas a la mezcla de jugo de maracuyá y piña, en combinación con los subproductos de estas frutas, que por lo general en la industria se consideran desechos, son significativamente positivos. Al comparar el aumento porcentual de los siguientes nutrientes en las muestras con tratamiento y sin tratamiento, los resultados numéricos son los siguientes: proteína $60 \%$, grasa $13 \%$, fibra cruda $522 \%$, cenizas $18 \%$; sin embargo, los carbohidratos digeribles disminuyeron en un $22,2 \%$.
El aumento en más de un $500 \%$ en fibras se debe a que aquellas macromoléculas que conforman la pared celular y que son insolubles y además no digeribles, son parcialmente digeridas por el catalizador biológico, convirtiéndolas en fibras de mucho menor tamaño, que siguen siendo no digeribles por el cuerpo humano, pero tienen una acción que mejora el funcionamiento del sistema digestivo, favoreciendo el aumento del peristaltismo, evitando acumulación de alimentos difíciles de digerir en los intersticios en la pared de los intestinos, previniendo enfermedades infecciosas y algunos tipos de cáncer.

Dentro de estas fibras, están cuantificadas también las pectinas, que se ha encontrado que ejercen una acción favorable en el control de las lipoproteínas de baja densidad (LDL), lo que permite controlar el entaponamiento de las arterias y una baja irrigación a órganos vitales, disminuyendo el riesgo de ciertas enfermedades como la hipertensión arterial y aumentando la calidad de vida del ser humano. Además, favorece su uso en pacientes diabéticos en vista de que son carbohidratos que dan saciedad y no se absorben. A nivel industrial las pectinas tienen varias aplicaciones, en el caso que nos compete le aporta estabilidad en fibra y pulpa a la bebida, además, de buena textura y brillo al producto.

Otro resultado que llama la atención favorablemente es la disminución de los carbohidratos digeribles, los cuales disminuyeron en un $22 \%$ después del tratamiento, esto hace al producto más amigable desde el punto de vista nutricional y de la salud, tomando en cuenta que los carbohidratos de este tipo son relativamente económicos, de fácil acceso, y la oferta mercadológica es más agresiva. Sin embargo, este resultado puede generar la oferta de un jugo nutricionalmente más equilibrado, de bajo nivel de carbohidratos digeribles.

El $13 \%$ de aumento de la grasa bruta que se puede observar, por lo general se pierde en lo que la industria 
estima como desecho después del procesamiento de las frutas. Esto así, porque esas grasas se encuentran dentro de las células vegetales y atrapadas en los intersticios de la macromolécula que forman la pared celular. Al aplicarle las biomoléculas con la finalidad de quitarle segmentos de fibras a la pared celular, la misma se debilita estallando, producto de la presión osmótica y liberando todo su contenido interno. Se estima que este incremento de las grasas en el producto, está relacionado con el aumento de ácidos grasos insaturados y otras sustancias liposolubles como los betacarotenos (precursor de la vitamina A), el cual tiene varias propiedades nutracéuticas como es su poder antioxidante, además puede evitar la ceguera nocturna, produce renovación de los epitelios y le da un buen respaldo al sistema inmunológico.

En la tabla 6 están plasmados los resultados de la sedimentación de las muestras tratadas con biomoléculas. En el mismo se observa que en las cuatro áreas en que las muestras fueron expuestas durante 5 horas, tal como se aprecia en las figuras 3, 4, 5 y 6; las muestras tratadas presentan mayor estabilidad, es decir, menor sedimentación que las muestras que no fueron tratadas, demostrando que en un área por debajo de $18{ }^{\circ} \mathrm{C}$, las muestras se mantienen estables y aún en temperaturas no aptas para la conservación de este tipo de productos (25 y $30{ }^{\circ} \mathrm{C}$, respectivamente), las muestras con tratamiento presentan mayor estabilidad que las muestras sin tratamiento.

En la tabla 7 se presentan los resultados de la sedimentación, transcurrido un tiempo de 24 horas de la preparación de las muestras y exposición a las diferentes temperaturas. Como se puede observar, en las figuras 7 y 8 , la muestra con tratamiento continúa manteniendo su estabilidad, al contrario de la muestra que no fue tratada con catalizador, que su sedimentación va en aumento. En los resultados de la sedimentación de las áreas 3 $\left(25^{\circ} \mathrm{C}\right)$ y $4\left(30{ }^{\circ} \mathrm{C}\right)$ respectivamente, tal como se aprecia en las figuras 9 y 10 , se puede observar que tanto las muestras tratadas como las muestras sin tratamiento, presentan separación en uno de su respectivo duplicado, evidenciando esto que la exposición durante 24 horas a estas temperaturas, provocan pérdida de la estabilidad de la mezcla.

En la tabla 8 se muestran los resultados de la sedimentación, transcurrido un tiempo de 48 horas de la preparación de las muestras y exposición a las diferentes temperaturas. Como se puede observar, en la figura 11, la muestra con tratamiento continúa manteniendo su estabilidad, al contrario de la muestra que no fue tratada con catalizador, que su sedimentación siguió aumentando. En los resultados de la sedimentación de las áreas $2\left(17^{\circ} \mathrm{C}\right)$, $3\left(25^{\circ} \mathrm{C}\right)$ y $4\left(30^{\circ} \mathrm{C}\right)$, tal como se aprecia en las figuras 12,13 y 14 , se puede observar que tanto las muestras tratadas como las muestras sin tratamiento, presentan separación en uno de su respectivo duplicado, unos en la parte superior y otros en el fondo del envase, evidenciando esto que la exposición durante 48 horas a estas temperaturas, provocan pérdida de la estabilidad de la mezcla.

\section{Conclusión}

Bajo las condiciones en que se realizaron los experimentos, la reacción de la cinética enzimática tiene una duración de 1 hora 55 minutos.

D El proceso de desagregación de la pulpa tuvo como resultado un jugo estable en su turbidez con un color y olor bastante acentuado, relacionado con las frutas que se han utilizado para preparar el mismo.

$>$ El producto tratado con el biocatalizador tiene mayores propiedades nutracéuticas que el jugo sin tratamiento.

El jugo tratado con el biocatalizador es más estable que el jugo sin tratamiento. 


\section{Referencias}

1. Vega Acosta AC. Plan de negocio de una bebida refrescante saludable, a base de maracuyá y stevia, en la ciudad de Guayaquil. Repositorio Universidad de Guayaquil. 2016.

2. de Queiroz MdSR, Janebro DI, da Cunha MAL, Medeiros JS, Sabaa-Srur AU, Diniz MFFM, et al. Effect of the yellow passion fruit peel flour (Passiflora edulis f. flavicarpa deg.) in insulin sensitivity in type 2 diabetes mellitus patients. Nutrition Journal. 2012 Octubre; 11(89).

3. López Vargas JH, Fernández López J, Pérez Álvarez JA, Viuda Martos M. Chemical, physico-chemical, technological, antibacterial and antioxidant properties of dietary fiber powder obtained from yellow passion fruit (Passiflora edulis var. flavicarpa) co-products. Food Research International. 2013;51(2).

4. Díaz LS, Padilla C, Carolina S. Identificación del Principal Pigmento Presente en la Cáscara del Maracuyá Púrpura (Passiflora edulis). Scielo Chile. 2006;17(06).

5. Sánchez WF, Murillo E, Méndez JJ. Potencial antioxidante de residuos agroindustriales de tres frutas de alto consumo en el Tolima. Scientia et technica. 2010;3(46).

6. Zeraik ML, Yariwake JH, Wauters JN, Tits M, Angenot L. Analysis of passion fruit rinds (Passiflora edulis): isoorientin quantification by HPTLC and evaluation of antioxidant (radical scavenging) capacity. Química Nova. 2012;35(3).

7. Ancona DB, Flores VP, Guerrero LAC. Fibra dietética y sus beneficios en la alimentación. Revista de la Universidad Autónoma de Yucatán. 2003 Cuarto trimestre;(227).
8. Matos Chamorro A, Chambilla Mamani E. Importancia de la Fibra Dietética, sus Propiedades Funcionales en la Alimentación Humana y en la Industria Alimentaria. Revista de Investigación en Ciencia y Tecnología de Alimentos. 2010;1(1).

9. Ramos Alessandra Teixeira CMAL,SSAUO,PVCF,CAA,DMdFMea. Use of Passiflora edulis f. flavicarpa on cholesterol reduction. Revista Brasileira de Farmacognosia. Revista Brasileira de Farmacognosia. 2007 diciembre;17(4).

10. Wheateer C. Zumos para una vida sana Barcelona: MC Produció Editorial; 2004.

11. Durán V, Honores M, P. C. Obtención de pectina en polvo a partir de la cáscara de maracuyá (passiflora edulis). DSpace en Spol; 2012.

12. Páez G, Marín M, Mármol Z, Ferrer J, D’Addosio R. Obtención y caracterización de pectina a partir de la cáscara de parchita (Passiflora edulis f. flavicarpa Degener). Revista de la Facultad de Agronomía de la Universidad del Zulia. 2005;22(3).

13. Rivadeneira M, Cáceres P. Extracción de pectina líquida a partir de cáscaras de maracuyá (passiflora edulis) y su aplicación en el desarrollo de un producto de humedad intermedia. DSpace en ESPOL; 2010 abril.

14. Vargas J, López H. Caracterización de coproductos de la industria del maracuyá (passiflora var. Flavicarpa) y su aplicación a productos cárnicos. Dialnet; 2013.

15. do Espírito Santo AP, Perego P, Converti A, Oliveira MN. Influence of milk type and addition of passion fruit peel powder on fermentation kinetics, texture profile and bacterial viability in probiotic yoghurts. LWT-Food Science and Technology. 2012 Julio;47(2). 
16. A.O.A.C. Official Methods of Analysis of AOAC International. AOAC International. Gaithersburg, M.D. 16th ed. Gaithersburg, M.D.: AOAC International; 1996.
17. Worthington Biochemical Corporation. Celullase. In von, editor. Enzyme and related biochemicals Hand Book. New Jersey, U.S.A.: Worthington Biochemical Corp.; 1993. p. 81-85. 